\title{
HOMOLOGY CLASSES WHICH ARE REPRESENTED BY GRAPH LINKS
}

\author{
KOICHI YANO
}

\begin{abstract}
We give a necessary and sufficient condition for a one-dimensional homology class of a graph manifold to be represented by a graph link.
\end{abstract}

The purpose of this paper is to give a necessary and sufficient condition for a one-dimensional homology class of a graph manifold to be represented by a graph link. For this, we define the Jaco-Shalen-Johannson complex of a graph manifold (§1), using the so-called torus decomposition theorem due to Jaco-Shalen and Johannson (see also Waldhausen [2]), and the main result is stated as follows (\$2):

THEOREM. Let $M$ be a graph manifold prime to $S^{1} \times S^{2}$ and $\rho: M \rightarrow \mathscr{C}_{M}$ the natural map to the Jaco-Shalen-Johannson complex of $M$. Then an element $\alpha$ of $H_{1}(M ; \mathbf{Z})$ can be represented by a graph link if and only if $\rho_{*}(\alpha)=0$ in $H_{1}\left(\mathscr{C}_{M} ; \mathbf{Z}\right)$.

If the ambient manifold $M$ is not prime to $S^{1} \times S^{2}$, the homotopy class of the map $\rho: M \rightarrow \mathscr{C}_{M}$ is not unique in general, and this makes the statement complicated (§3). The proof of these results is based on the study of global graph links in [3].

1. Preliminaries. Throughout this paper, manifolds are compact, oriented, of dimension three and with toral boundary, and links are oriented. A manifold $M$ is a graph manifold if there is a family of disjointly embedded tori in $M$ such that each connected component of the manifold obtained by cutting $M$ along these tori is the total space of an $S^{1}$-bundle over a surface. A link is called a graph link if its exterior is a graph manifold. We say that a manifold is prime to $S^{1} \times S^{2}$ if its prime decomposition does not contain $S^{1} \times S^{2}$. See Jaco [1] and Yano [3] for other terminology.

To state our result, we need the following theorem due to Jaco-Shalen and Johannson (see Jaco [1] and also Waldhausen [2]).

THEOREM 1.1 (JACO-SHALEN, JoHANNSON). Let $M$ be a Haken manifold which is either closed or with incompressible boundary. Then there exists a unique Seifert submanifold $\Sigma \subset M$ up to ambient isotopy such that

(1) $\Sigma$ is maximal, and

(2) if $\Sigma^{\prime}$ is a Seifert manifold pair distinct from $\left(S^{3}, \varnothing\right),\left(S^{2} \times S^{1}, \varnothing\right),\left(D^{2} \times\right.$ $\left.I, \partial D^{2} \times I\right)$ or $\left(S^{1} \times D^{2}, \varnothing\right)$, then every nondegenerate map $f: \Sigma^{\prime} \rightarrow(M, \partial M)$ is homotopic to $f_{0}$ such that $f_{0}\left(\Sigma^{\prime}\right) \subset \Sigma$.

The $\Sigma$ above is called the characteristic Seifert manifold of $M$.

Received by the editors September 19, 1983 and, in revised form, March 14, 1984.

1980 Mathematics Subject Classification. Primary 57M99, 57N10.

Key words and phrases. Graph manifold, graph link, homology class, characteristic Seifert manifold.

(1985 American Mathematical Society $0002-9939 / 85 \$ 1.00+\$ .25$ per page 
For a graph manifold $M$, we define a 1-complex $\mathscr{C}_{M}$, called the Jaco-ShalenJohannson complex of $M$, as follows: For a Seifert manifold $M$ distinct from $\mathbf{R} P^{3} \# \mathbf{R} P^{3}$, we define $\mathscr{C}_{M}$ to be a point. Let $M$ be a Haken graph manifold and $\Sigma$ the characteristic Seifert manifold of $M$. Let $\Sigma_{1}, \ldots, \Sigma_{k}$ be the components of $\Sigma$ and $E_{1}, \ldots, E_{l}$ the components of $M-$ int $\Sigma$. Note that, by definition, each $E_{j}$ is diffeomorphic to $T^{2} \times I$. Then we put

$$
\mathscr{C}_{M}=\left\{v_{1}, \ldots, v_{k}, e_{1}, \ldots, e_{l}\right\},
$$

where $v_{i}$ is a vertex corresponding to $\Sigma_{i}$, and $e_{j}$ is a 1-cell corresponding to $E_{j}$, thus connecting $v_{i}$ and $v_{i^{\prime}}$ such that $\Sigma_{i} \cap E_{j} \neq \varnothing$ and $\Sigma_{i^{\prime}} \cap E_{j} \neq \varnothing$. In general, take a homeomorphism $h: M \rightarrow M_{1} \# \cdots \# M_{n}$ which gives a prime decomposition of $M$. Since each $M_{i}$ is either a Seifert manifold or a Haken graph manifold, there is already defined the Jaco-Shalen-Johannson complex $\mathscr{C}_{M_{i}}$. Then we put

$$
\mathscr{C}_{M}=\bigcup_{i=1}^{n} \mathscr{C}_{M_{i}} \cup\left\{\bar{e}_{1}, \ldots, \bar{e}_{n-1}\right\},
$$

where each $\bar{e}_{i}$ connects suitable vertices of $\mathscr{C}_{M_{i}}$ and $\mathscr{C}_{M_{i+1}}$. Although this definition (for nonirreducible manifolds) depends on the homeomorphism $h$ and the choice of vertices, the homotopy type of $\mathscr{C}_{M}$ is independent of them. For a complex $\mathscr{C}_{M}$ thus defined, there is a natural map $\rho: M \rightarrow \mathscr{C}_{M}$. If the manifold $M$ is prime to $S^{1} \times S^{2}$, then the homotopy class of $\rho$ does not depend on the $h$ above and the choice of vertices.

Finally we recall a result from [3] which is needed for the proof.

THEOREM 1.2. Every graph link is obtained from elementary graph links by a finite number of operations of disjoint union, adding a cable knot of some component, replacing one component by its cable knot, link connected sum, and self link connected sum.

Link connected sum and self link connected sum are indicated by Figure 1. Note that if $L \subset M$ is an elementary graph link, then the ambient manifold $M$ is either irreducible or diffeomorphic to $S^{1} \times S^{2}$, and each component of $L$ is contained in a nondegenerate Seifert submanifold of $M$. (See [3] for the precise definition.)

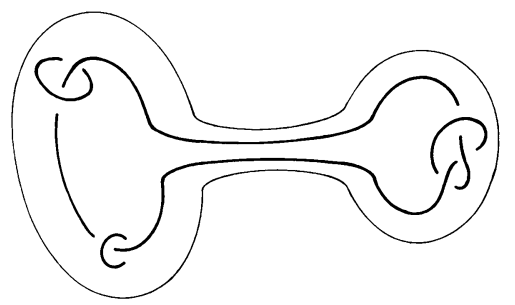

Link connected sum

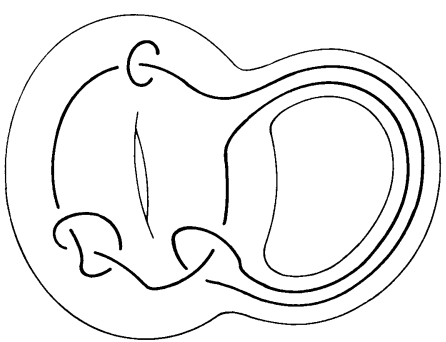

Self link connected sum

FIGURE 1 
2. Graph manifolds prime to $S^{1} \times S^{2}$. In this section we prove the main result.

THEOREM 2.1. Let $M$ be a graph manifold prime to $S^{1} \times S^{2}$ and $\rho: M \rightarrow \mathscr{C}_{M}$ the natural map to the Jaco-Shalen-Johannson complex of $M$. Then an element $\alpha$ of $H_{1}(M ; \mathbf{Z})$ can be represented by a graph link if and only if $\rho_{*}(\alpha)=0$ in $H_{1}\left(\mathscr{C}_{M} ; \mathbf{Z}\right)$.

First we deal with Seifert manifolds.

Proposition 2.2. Let $M$ be a Seifert manifold. Then every element of $H_{1}(M ; \mathbf{Z})$ can be represented by a graph link.

Proof. Let $\hat{M}$ be the manifold obtained from $M$ by deleting tubular neighborhoods of singular fibres and one regular fibre. Then the natural inclusion map $\iota: \hat{M} \rightarrow M$ induces an onto map $\iota_{*}: H_{1}(\hat{M} ; \mathbf{Z}) \rightarrow H_{1}(M ; \mathbf{Z})$. Therefore we can assume without loss of generality that $M$ has no singular fibre and the boundary of $M$ is nonempty. First suppose that the Seifert fibring of $M$ is not twisted. Then we have $M \cong F \times S^{1}$ for a surface $F$. The proof of the following lemma is immediate and is omitted.

LEMMA 2.3. Let $F$ be a surface possibly with boundary and $\alpha$ be a one-dimensional homology class of $F$. Then there exists a family of disjoint simple closed curves $c_{1}, \ldots, c_{n}$ on $F$ and integers $a_{1}, \ldots, a_{n}$ such that $\alpha=\sum_{i} a_{i}\left[c_{i}\right]$.

Let $\alpha=\alpha_{1}+\alpha_{2}$ under the identification $H_{1}(M ; \mathbf{Z}) \cong H_{1}(F ; \mathbf{Z}) \oplus H_{1}\left(S^{1} ; \mathbf{Z}\right)$. Applying Lemma 2.3 to $\alpha_{1}$, we have a family of disjoint curves $c_{1}, \ldots, c_{n}$ on $F$. Since each $c_{i} \times\{*\}$ lies on the torus $c_{i} \times S^{1}$, the link $\bigcup_{i=1}^{n} c_{i} \times\{*\} \cup\{* *\} \times S^{1}$ is a graph link in $M$. Thus we obtain a required link from this one by replacing each component by its suitable cable knot.

The proof for the case of twisted Seifert fibring is reduced to the above case, since the twisted $S^{1}$-bundle over the Möbius band has a Seifert fibred structure over $D^{2}$. This completes the proof.

Proof of Theorem 2.1. By Proposition 2.2 and the Mayer-Vietoris exact sequence, we see that every element $\alpha$ of $H_{1}(M ; \mathbf{Z})$ with $\rho_{*}(\alpha)=0$ in $H_{1}\left(\mathscr{C}_{M} ; \mathbf{Z}\right)$ can be represented by a graph link.

Now we prove the converse. Let $k$ be an elementary graph knot in $M$. By definition, the ambient manifold $M$ is irreducible and the knot $k$ is contained in a nondegenerate Seifert submanifold $\Sigma^{\prime}$ of $M$. By Theorem 1.1, the map $\Sigma^{\prime} \rightarrow M \rightarrow \mathscr{C}_{M}$ is homotopic to a constant map, and therefore $\rho_{*}([k])=0$ in $H_{1}\left(\mathscr{C}_{M} ; \mathbf{Z}\right)$. This, together with induction on the number of operations in Theorem 1.2, implies the required result.

3. Graph manifolds not prime to $S^{1} \times S^{2}$. In this section we deal with manifolds not prime to $S^{1} \times S^{2}$. We begin with definitions and lemmas.

For an element $\gamma$ of $H_{1}\left(\#_{n=1}^{k} S^{1} \times S^{2} ; \mathbf{Z}\right)$, we put $m(\gamma)$ to be the maximal integer $m$ such that there exists an element $\gamma^{\prime}$ satisfying $\gamma=m \gamma^{\prime}$ (we put $m(0)=0$ ). We say that $\gamma$ is primitive if $m(\gamma)=1$. 


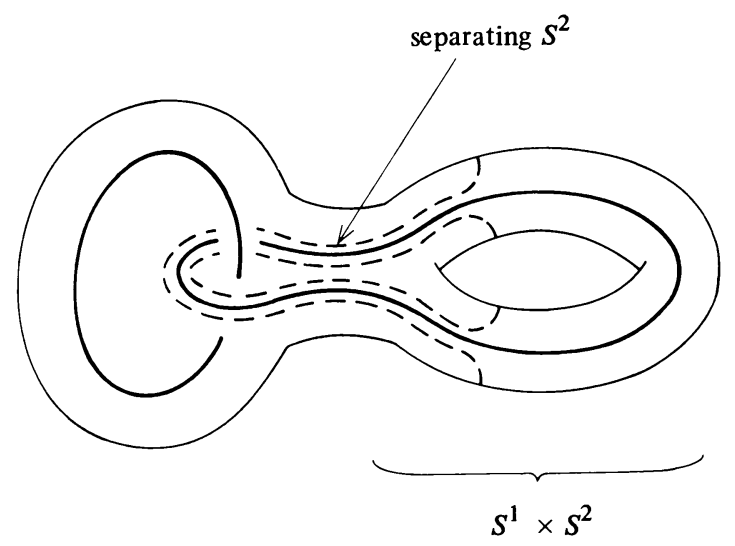

FIGURE 2

LEMMA 3.1. Let $k_{0}$ be an elementary graph knot in $S^{1} \times S^{2}$ i.e., $k_{0}=S^{1} \times\{*\}$. Let $L_{1}$ and $k_{1}$ be any link and any knot disjoint in a manifold $N$. Then there exists a homeomorphism $h: N \# S^{1} \times S^{2} \rightarrow N \# S^{1} \times S^{2}$ satisfying $h\left(L_{1}\right)=L_{1}$ and $h\left(k_{0}\right)=$ $k_{1} \# k_{0}$.

Proof. There is an embedded sphere in $N \# S^{1} \times S^{2}$ which separates $L_{1}$ and $k_{1} \# k_{0}$ as in Figure 2.

LEMMA 3.2. Let $\gamma$ be a primitive element of $H_{1}\left(\#_{n=1}^{k} S^{1} \times S^{2} ; \mathbf{Z}\right)$ and $k_{0}$ be an elementary graph knot in $S^{1} \times S^{2} \subset \#_{n=1}^{k} S^{1} \times S^{2}$. Then there exists a homeomorphism h: $\#_{n=1}^{k} S^{1} \times S^{2} \rightarrow \#_{n=1}^{k} S^{1} \times S^{2}$ such that $\left[h\left(k_{0}\right)\right]=\gamma$.

Proof. Let $\alpha_{1}, \ldots, \alpha_{k}$ be the canonical basis of $H_{1}\left(\#_{n=1}^{k} S^{1} \times S^{2} ; \mathbf{Z}\right)$. By Lemma 3.1 , there is a homeomorphism $h$ of $\#_{n=1}^{k} S^{1} \times S^{2}$ such that

$$
h_{*}\left(\alpha_{1}\right)=\alpha_{1}+\sum_{j \neq 1} a_{j} \alpha_{j} \text { and } h_{*}\left(\alpha_{l}\right)=\alpha_{l}, \quad l \neq 1,
$$

for any integers $a_{2}, \ldots, a_{k}$, and obviously permutations of $\left\{\alpha_{1}, \ldots, \alpha_{k}\right\}$ are attained by homeomorphisms. Any isomorphism of $H_{1}\left(\#_{n=1}^{k} S^{1} \times S^{2} ; \mathbf{Z}\right)$ is given by a combination of these ones and thus is induced by a homeomorphism of

$$
\underset{n=1}{\prod_{n}} S^{1} \times S^{2}
$$

This implies the required result.

Let $M$ be a graph manifold which has exactly $k$ prime factors diffeomorphic to $S^{1} \times S^{2}$. Fix a homeomorphism $h: M \cong N \# \#_{n=1}^{k} S^{1} \times S^{2}$, where $N$ is prime to $S^{1} \times S^{2}$. Then there is an exact sequence

$$
0 \rightarrow H_{1}(N ; \mathbf{Z}) \stackrel{i_{*}}{\rightarrow} H_{1}(M ; \mathbf{Z}) \stackrel{p_{*}}{\rightarrow} H_{1}\left(\underset{n=1}{\stackrel{k}{\#}} S^{1} \times S^{2} ; \mathbf{Z}\right) \rightarrow 0 .
$$


Since Image $i_{*}=\operatorname{Ker} p_{*}$ is given by

$$
\operatorname{Ker} p_{*}=\left\{\alpha \in H_{1}(M ; \mathbf{Z}) ; \alpha \circ\left[S^{2}\right]=0 \text { for every embedded } S^{2} \subset M\right\},
$$

this exact sequence is unique up to homeomorphisms of $N$ and $\#_{n=1}^{k} S^{1} \times S^{2}$. Let $s_{h}: H_{1}(M ; \mathbf{Z}) \rightarrow H_{1}(N ; \mathbf{Z})$ be the splitting defined by the $h$ above, and $\rho_{N}: N \rightarrow \mathscr{C}_{N}$ the natural map to the Jaco-Shalen-Johannson complex of $N$. We define a subset $G(M)$ of $H_{1}(M ; \mathbf{Z})$ by

$$
G(M)=\left\{\alpha \in H_{1}(M ; \mathbf{Z}) ; \rho_{N^{*}}\left(s_{h}(\alpha)\right) \text { is divisible by } m\left(p_{*}(\alpha)\right)\right\} .
$$

It is easy to see that this definition does not depend on the choice of a homeomorphism $h$. Now the result of this section is

THeOReM 3.3. Let $M$ be a graph manifold. Then an element $\alpha$ of $H_{1}(M ; \mathbf{Z})$ can be represented by a graph link if and only if $\alpha$ is in $G(M)$.

Proof. Take a homeomorphism $h: M \rightarrow N \# \#_{n=1}^{k} S^{1} \times S^{2}$, where $N$ is prime to $S^{1} \times S^{2}$. Then there is an identification

$$
H_{1}(M ; \mathbf{Z}) \cong H_{1}(N ; \mathbf{Z}) \oplus H_{1}\left(\underset{n=1}{k} S^{1} \times S^{2} ; \mathbf{Z}\right)
$$

Let $\alpha=\beta+\gamma$ under this identification and suppose that $\alpha \in G(M)$. Then we can put $\beta=\beta_{0}+m \beta_{1}$ and $\gamma=m \bar{\gamma}$ such that $\rho_{N^{*}}\left(\beta_{0}\right)=0$ and $\bar{\gamma}$ is primitive. By Theorem 2.1, we have a graph link $L$ which represents $\beta_{0}$. Take a knot $k_{1}$ of $N$ which is disjoint from $L$ and represents $\beta_{1}$. Then, by Lemmas 3.1 and 3.2, we have a homeomorphism

$$
h: N \# \underset{n=1}{\#} S^{1} \times S^{2} \rightarrow N \# \underset{n=1}{\#} S^{1} \times S^{2}
$$

such that $h(L)=L$ and $\left[h\left(k_{0}\right)\right]=\beta_{1}+\bar{\gamma}$, where $k_{0}$ is an elementary graph knot in $S^{1} \times S^{2} \subset \#_{n=1}^{k} S^{1} \times S^{2}$. Since $L \cup k_{0}$ is a graph link, we obtain a required link from $h\left(L \cup k_{0}\right)$ by replacing $h\left(k_{0}\right)$ by its cable knot.

For the converse, it suffices to show the following

Proposition 3.4. Let $M$ be as above and $L$ a graph link in $M$. Then there exists a homeomorphism $h: M \rightarrow N \# \#_{n=1}^{k} S^{1} \times S^{2}$ such that every component $k$ of $L$ satisfies $\rho_{N^{*}}\left(s_{h}([k])\right)=0$.

Proof. If $L$ is an elementary graph link, then the ambient manifold $M$ is either irreducible or diffeomorphic to $S^{1} \times S^{2}$ and thus the result follows from Theorem 2.1. Using this and induction on the number of operations in Theorem 1.2, we have this proposition, and this completes the proof of Theorem 3.3.

REMARK. By the proof, it is easy to see the following: For a graph manifold $M$, there exists an integer $n(M)$ such that every homology class $\alpha \in G(M)$ can be represented by a graph link, the number of components of which is at most $n(M)$. 


\section{REFERENCES}

1. W. H. Jaco, Lectures on three-manifolds topology, CBMS Regional Conf. Ser. Math., no. 43, Amer. Math. Soc., Providence, R. I., 1980.

2. F. Waldhausen, Eine Klasse von 3-dimensionalen Mannigfaltigkeiten. I; II, Invent. Math. 3 (1967), 308-333; 4 (1967), 87-117.

3. K. Yano, The support of global graph links, J. Math. Soc. Japan (to appear).

Department of Mathematics, Faculty of Science, University of Tokyo, Hongo, Tokyo, 113 JAPAN 